\title{
Numerical method on drug release from Nanoparticles using CFD.
}

\begin{abstract}
Nowadays, there are many ways to administer the huge variety of drugs that are on the market. A drug is a chemical substance, that when applied to a living creature, alters its body function. Many drugs are used daily, such as caffeine in coffee and aspirin for pain. A route of drug administration in pharmacology and toxicology is the path by which a drug is brought into contact with the body. The most critical issue is some drugs are not significantly absorbed into the bloodstream. There are thousands of questions on the drug diffusion in the bloodstream and the most common issue is how long will these micro particles drug be released from the tablet? Thus, to identify the rate of the drug release and to control the drug release in our body is important, where to meet the target and not to become a waste. The Computational Fluid Dynamics method was used to investigate the drug design and diffusion profiles with time during the process of degradation and diffusion in water.
\end{abstract}

Keyword: Drug; CFD; Nanoparticles; Diffusio; Release; Profile. 